\title{
Robust Model Predictive Control Design
}

\author{
Vojtech Veselý and Danica Rosinová \\ Institute for Control and Industrial Informatics, Faculty of Electrical Engineering and \\ Information Technology, Slovak University of Technology, Ilkovičova 3, 81219 Bratislava \\ Slovak Republic
}

\section{Introduction}

Model predictive control (MPC) has attracted notable attention in control of dynamic systems and has gained the important role in control practice. The idea of MPC can be summarized as follows, (Camacho \& Bordons, 2004), (Maciejovski, 2002), (Rossiter, 2003) :

- Predict the future behavior of the process state/output over the finite time horizon.

- Compute the future input signals on line at each step by minimizing a cost function under inequality constraints on the manipulated (control) and/or controlled variables.

- Apply on the controlled plant only the first of vector control variable and repeat the previous step with new measured input/state/output variables.

Therefore, the presence of the plant model is a necessary condition for the development of the predictive control. The success of MPC depends on the degree of precision of the plant model. In practice, modelling real plants inherently includes uncertainties that have to be considered in control design, that is control design procedure has to guarantee robustness properties such as stability and performance of closed-loop system in the whole uncertainty domain. Two typical description of uncertainty, state space polytope and bounded unstructured uncertainty are extensively considered in the field of robust model predictive control. Most of the existing techniques for robust MPC assume measurable state, and apply plant state feedback or when the state estimator is utilized, output feedback is applied. Thus, the present state of robustness problem in MPC can be summarized as follows:

Analysis of robustness properties of MPC.

(Zafiriou \& Marchal, 1991) have used the contraction properties of MPC to develop necessarysufficient conditions for robust stability of MPC with input and output constraints for SISO systems and impulse response model. (Polak \& Yang, 1993) have analyzed robust stability of MPC using a contraction constraint on the state.

MPC with explicit uncertainty description.

(Zheng \& Morari, 1993), have presented robust MPC schemes for SISO FIR plants, given uncertainty bounds on the impulse response coefficients. Some MPC consider additive type of uncertainty, (delaPena et al., 2005) or parametric (structured) type uncertainty using CARIMA model and linear matrix inequality, (Bouzouita et al., 2007). In (Lovas et al., 2007), for openloop stable systems having input constraints the unstructured uncertainty is used. The robust stability can be established by choosing a large value for the control input weighting matrix $R$ in the cost function. The authors proposed a new less conservative stability test for determining a sufficiently large control penalty $R$ using bilinear matrix inequality (BMI). In (Casavola 
et al., 2004), robust constrained predictive control of uncertain norm-bounded linear systems is studied. The other technique- constrained tightening to design of robust MPC have been proposed in (Kuwata et al., 2007). The above approaches are based on the idea of increasing the robustness of the controller by tightening the constraints on the predicted states.

The mixed $\mathrm{H}_{2} / \mathrm{H}_{\infty}$ control approach to design of MPC has been proposed by (Orukpe et al., 2007).

Robust constrained MPC using linear matrix inequality (LMI) has been proposed by (Kothare et al., 1996), where the polytopic model or structured feedback uncertainty model has been used. The main idea of (Kothare et al., 1996) is the use of infinite horizon control laws which guarantee robust stability for state feedback. In (Ding et al., 2008) output feedback robust MPC for systems with both polytopic and bounded uncertainty with input/state constraints is presented. Off-line, it calculates a sequence of output feedback laws based on the state estimators, by solving LMI optimization problem. On-line, at each sampling time, it chooses an appropriate output feedback law from this sequence. Robust MPC controller design with one step ahead prediction is proposed in (Veselý \& Rosinová , 2009). The survey of optimal and robust MPC design can be consulted in (Mayne et al., 2000). Some interesting results for nonlinear MPC are given in (Janík et al., 2008).

In MPC approach generally, control algorithm requires solving constrained optimization problem on-line (in each sampling period). Therefore on-line computation burden is significant and limits practical applicability of such algorithms to processes with relatively slow dynamics. In this chapter, a new MPC scheme for an uncertain polytopic system with constrained control is developed using model structure introduced in (Veselý et al., 2010). The main contribution of the first part of this chapter is that all the time demanding computations of output feedback gain matrices are realized off-line ( for constrained control and unconstrained control cases). The actual value of control variable is obtained through simple on-line computation of scalar parameter and respective convex combination of already computed matrix gains. The developed control design scheme employs quadratic Lyapunov stability to guarantee the robustness and performance (guaranteed cost) over the whole uncertainty domain.

The first part of the chapter is organized as follows. A problem formulation and preliminaries on a predictive output/state model as a polytopic system are given in the next section. In Section 1.2, the approach of robust output feedback predictive controller design using linear matrix inequality is presented. In Section 1.3, the input constraints are applied to LMI feasible solution. Two examples illustrate the effectiveness of the proposed method in the Section 1.4. The second part of this chapter addresses the problem of designing a robust parameter dependent quadratically stabilizing output/state feedback model predictive control for linear polytopic systems without constraints using original sequential approach. For the closed-loop uncertain system the design procedure ensures stability, robustness properties and guaranteed cost. Finally, conclusions on the obtained results are given.

Hereafter, the following notational conventions will be adopted: given a symmetric matrix $P=P^{T} \in R^{n \times n}$, the inequality $P>0(P \geq 0)$ denotes matrix positive definiteness (semidefiniteness). Given two symmetric matrices $P, Q$, the inequality $P>Q$ indicates that $P-Q>0$. The notation $x(t+k)$ will be used to define, at time $t, \mathrm{k}$-steps ahead prediction of a system variable $x$ from time $t$ onwards under specified initial state and input scenario. $I$ denotes the identity matrix of corresponding dimensions. 


\subsection{Problem formulation and preliminaries}

Let us start with uncertain plant model described by the following linear discrete-time uncertain system with polytopic uncertainty domain

$$
\begin{gathered}
x(t+1)=A(\alpha) x(t)+B(\alpha) u(t) \\
y(t)=C x(t)
\end{gathered}
$$

where $x(t) \in R^{n}, u(t) \in R^{m}, y(t) \in R^{l}$ are state, control and output variables of the system, respectively; $A(\alpha), B(\alpha)$ belong to the convex set

$$
\begin{gathered}
S=\left\{A(\alpha) \in R^{n \times n}, B(\alpha) \in R^{n \times m}\right\} \\
\left\{A(\alpha)=\sum_{j=1}^{N} A_{j} \alpha_{j} \quad B(\alpha)=\sum_{j=1}^{N} B_{j} \alpha_{j}, \alpha_{j} \geq 0\right\}, j=1,2 \ldots N, \sum_{j=1}^{N} \alpha_{j}=1
\end{gathered}
$$

Matrices $A_{i}, B_{i}$ and $C$ are known matrices with constant entries of corresponding dimensions. Simultaneously with (1) we consider the nominal model of system (1) in the form

$$
x(t+1)=A_{o} x(t)+B_{o} u(t) \quad y(t)=C x(t)
$$

where $A_{0}, B_{0}$ are any constant matrices from the convex bounded domain $S$ (2). The nominal model (3) will be used for prediction, while (1) is considered as real plant description providing plant output. Therefore in the robust controller design we assume that for time $t$ output $y(t)$ is obtained from uncertain model (1), predicted outputs for time $t+1, \ldots t+N_{2}$ will be obtained from model prediction, where the nominal model (3) is used. The predicted states and outputs of the system (1) for the instant $t+k, k=1,2, \ldots N_{2}$ are given by

- $\mathrm{k}=1$

$$
x(t+2)=A_{o} x(t+1)+B_{o} u(t+1)=A_{o} A(\alpha) x(t)+A_{o} B(\alpha) u(t)+B_{o} u(t+1)
$$

- $\mathrm{k}=2$

$$
x(t+3)=A_{o}^{2} A(\alpha) x(t)+A_{o}^{2} B(\alpha) u(t)+A_{o} B_{o} u(t+1)+B_{o} u(t+2)
$$

- for $\mathrm{k}$

$$
x(t+k+1)=A_{o}^{k} A(\alpha) x(t)+A_{o}^{k} B(\alpha) u(t)+\sum_{i=0}^{k-1} A_{o}^{k-i-1} B_{o} u(t+1+i)
$$

and corresponding output is

$$
y(t+k)=C x(t+k)
$$

Consider a set of $k=0,1,2, \ldots, N_{2}$ state/output model predictions as follows

$$
z(t+1)=A_{f}(\alpha) z(t)+B_{f}(\alpha) v(t), \quad y_{f}(t)=C_{f} z(t)
$$

where

$$
\begin{gathered}
z(t)^{T}=\left[x(t)^{T} \ldots x\left(t+N_{2}\right)^{T}\right], v(t)^{T}=\left[u(t)^{T} \ldots u\left(t+N_{u}\right)^{T}\right] \\
y_{f}(t)^{T}=\left[y(t)^{T} \ldots y\left(t+N_{2}\right)^{T}\right]
\end{gathered}
$$

and

$$
B_{f}(\alpha)=\left[\begin{array}{cccc}
B(\alpha) & 0 & \ldots & 0 \\
A_{o} B(\alpha) & B_{0} & \ldots & 0 \\
\ldots & \ldots & \ldots & 0 \\
A_{o}^{N_{2}} B(\alpha) & A_{o}^{N_{2}-1} B_{o} & \ldots & A_{o}^{N_{2}-N_{u}} B_{o}
\end{array}\right]
$$




$$
A_{f}(\alpha)=\left[\begin{array}{cccc}
A(\alpha) & 0 & \ldots & 0 \\
A_{0} A(\alpha) & 0 & \ldots & 0 \\
\ldots & \ldots & \ldots & \ldots \\
A_{o}^{N_{2}} A(\alpha) & 0 & \ldots & 0
\end{array}\right], C_{f}=\left[\begin{array}{cccc}
C & 0 & \ldots & 0 \\
0 & C & \ldots & 0 \\
\ldots & \ldots & \ldots & \ldots \\
0 & 0 & \ldots & C
\end{array}\right]
$$

where $N_{2}, N_{u}$ are output and control prediction horizons of model predictive control, respectively. Note that for output/state prediction in (6) one needs to put $A(\alpha)=A_{0}, B(\alpha)=B_{0}$. Matrices dimensions are $A_{f}(\alpha) \in R^{n\left(N_{2}+1\right) \times n\left(N_{2}+1\right)}, B_{f}(\alpha) \in R^{n\left(N_{2}+1\right) \times m\left(N_{u}+1\right)}$ and $C_{f} \in$ $R^{l\left(N_{2}+1\right) \times n\left(N_{2}+1\right)}$.

Consider the cost function associated with the system (6) in the form

$$
J=\sum_{t=0}^{\infty} J(t)
$$

where

$$
\begin{aligned}
J(t)=\sum_{k=0}^{N_{2}} x(t+k)^{T} Q_{k} x(t+k) & +\sum_{k=0}^{N_{u}} u(t+k)^{T} R_{k} u(t+k)= \\
& =z(t)^{T} Q z(t)+v(t)^{T} R v(t)
\end{aligned}
$$

$Q=$ blockdiag $\left\{Q_{i}\right\}_{i=0,1, \ldots N_{2}} R=$ blockdiag $\left\{R_{i}\right\}_{i=0,1, \ldots N_{u}}$

The problem studied in this part of chapter can be summarized as follows. Design the robust model predictive controller with output feedback and input constraints in the form

$$
v(t)=F y_{f}(t)=F C_{f} z(t)
$$

where $F^{T}=\left[F_{0}^{T} \ldots F_{N_{u}}^{T}\right], \quad F_{i}=\left[F_{i 0} \ldots F_{i N_{2}}\right], \quad i=0,1,2, \ldots N_{u}$ are the output feedback gain matrices which for given prediction horizon $\mathrm{N}_{2}$ and control horizon $N_{u}$ ensure the closed-loop system (13) stability, robustness and guaranteed cost.

$$
z(t+1)=\left(A_{f}(\alpha)+B_{f}(\alpha) F C_{f}\right) z(t)=A_{c}(\alpha) z(t)
$$

Definition 1. Consider the system (6). If there exists a control law $v(t)^{*}$ and a positive scalar $J^{*}$ such that the closed-loop system (13) is stable and the closed-loop cost function (10) value $J$ satisfies $J \leq J^{*}$ then $J^{*}$ is said to be the guaranteed cost and $v(t)^{*}$ is said to be the guaranteed cost control law for the system (6).

To guarantee closed-loop stability of uncertain system overall the whole uncertainty domain, the concept of quadratic stability is frequently used. That is, one Lyapunov function works for the whole uncertainty domain. Experience and analysis has shown that quadratic stability is rather conservative in many cases, therefore robust stability with parameter dependent Lyapunov function $P(\alpha)$ has been introduced by (Peaucelle et al., 2000). Using the concept of Lyapunov stability it is possible to formulate the following definition and lemma.

Definition 2. System (13) is robustly stable in the convex uncertainty domain with parameterdependent Lyapunov function $P(\alpha)$ if and only if there exists a matrix $P(\alpha)=P(\alpha)^{T}>0$ such that

$$
A_{c}(\alpha)^{T} P(\alpha) A_{\mathcal{c}}(\alpha)-P(\alpha)<0
$$

Lemma 1. (Rosinová et al., 2003), (Krokavec \& Filasová, 2003) Consider the closed-loop system (13) with control algorithm (12). Control algorithm (12) is the guaranteed cost control law if and only if there exists a positive definite matrix $P(\alpha)$ and matrix $F$ such that the following condition holds

$$
B_{e}=z(t)^{T}\left(A_{c}(\alpha)^{T} P(\alpha) A_{c}(\alpha)-P(\alpha)+Q+C_{f}^{T} F^{T} R F C_{f}\right) z(t) \leq 0
$$


where the first term of (15) $\Delta V(t)=z(t)^{T}\left(A_{\mathcal{c}}(\alpha)^{T} P(\alpha) A_{\mathcal{c}}(\alpha)-P(\alpha)\right) z(t)$ is the first difference of closed-loop system Lyapunov function $V(t)=z(t)^{T} P(\alpha) z(t)$. Moreover, summarizing (15) from initial time $t_{o}$ to $t \rightarrow \infty$ the following inequality is obtained

$$
-V\left(t_{0}\right)+J \leq 0
$$

Definition 1 and (16) imply

$$
J^{*} \leq V\left(t_{0}\right)
$$

Note, that as a receding horizon strategy is used, only $u(t)$ is sent to the real plant control, control inputs $u(t+k), k=0,1,2, \ldots, N_{u}$ are used for predictive outputs $y(t+k)$ calculation. According to (de Oliviera et al., 2000) there is no general and systematic way to formally determine $P(\alpha)$ in (15) as a function of $A_{c}(\alpha)$. Such a matrix $P(\alpha)$ is called the parameter dependent Lyapunov matrix (PDLM) and for particular structure of $P(\alpha)$ the inequality (15) defines the parameter dependent quadratic stability (PDQS). Formal approach to choose $P(\alpha)$ for real convex polytopic uncertainty (2) can be found in the references. One of the approaches is to take $P(\alpha)=P$, in this case if the solution is feasible the quadratic stability is obtained. Another possibility $P(\alpha)=\sum_{i=1}^{N} P_{i} \alpha_{i}, \sum_{i=1}^{N} \alpha_{i}=1, P_{i}=P_{i}^{T}>0$ gives the parameter dependent quadratic stability (PDQS). To decrease the conservatism of PDQS arising from affine parameter dependent Lyapunov function (PDLF), recently, the use of polynomial PDLF (PPDLF) has been proposed in different forms. For more details see (Ebihara et al., 2006).

\subsection{Robust model predictive controller design. Quadratic stability}

Robust MPC controller design which guarantees quadratic stability and guaranteed cost of closed-loop system is based on (15). Using Schur complement formula inequality (15) can be rewritten to following bilinear matrix inequality (BMI).

$$
\left[\begin{array}{ccc}
-P(\alpha)+Q & C_{f}^{T} F^{T} & A_{\mathcal{c}}(\alpha)^{T} \\
F C_{f} & -R^{-1} & 0 \\
A_{c}(\alpha) & 0 & -P(\alpha)^{-1}
\end{array}\right] \leq 0
$$

For the quadratic stability $P(\alpha)=P=P^{T}>0$ in (18). Using linearization approach for $P^{-1}$, de Oliviera et al. (2000), the following inequality can be derived

$$
-P^{-1} \leq Y_{k}^{-1}\left(P-Y_{k}\right) Y_{k}^{-1}-Y_{k}^{-1}=\operatorname{lin}\left(-P^{-1}\right)
$$

where $Y_{k}, k=1,2, \ldots$ in iteration process $Y_{k}=P$. We can recast bilinear matrix inequality (18) to the linear matrix inequality (LMI) using linearization (19). The following LMI is obtained for quadratic stability

$$
\left[\begin{array}{ccc}
-P+Q & C_{f}^{T} F^{T} & A_{f i}^{T}+C_{f}^{T} F^{T} B_{f i}^{T} \\
F C_{f} & -R^{-1} & 0 \\
A_{f i}+B_{f i} F C_{f} & 0 & \operatorname{lin}\left(-P^{-1}\right)
\end{array}\right] \leq 0 \quad i=1,2, \ldots N
$$

where

$$
A_{f}(\alpha)=\sum_{j=1}^{N} A_{f j} \alpha_{j} \quad B_{f}(\alpha)=\sum_{j=1}^{N} B_{f j} \alpha_{j}
$$

We can conclude that if the LMIs (20) are feasible with respect to $\varrho * I>P=P^{T}>0$ and matrix $F$ then the closed-loop system with control algorithm (12) is quadratically stable with 
guaranteed cost (17). Note that due to control horizon strategy only the first $m$ rows of matrix $F$ are used for real plant control, the other part of matrix $F$ serves for predicted output variables calculation. Parameter dependent or Polynomial parameter dependent quadratic stability approach to design robust MPC may decrease the conservatism of quadratic stability. In this case for PDQS we can use the approaches given in (Peaucelle et al., 2000), (Grman et al., 2005) and for (PPDLF) see (Ebihara et al., 2006).

\subsection{MPC design for input constraints}

In this subsection we propose the off-line calculation of two control gain matrices and using analogy to SVSC approach, (Adamy \& Fleming, 2004), we significantly reduce the computational effort for MPC suboptimal control with input constraints.

To design model predictive control (Adamy \& Fleming, 2004), (Camacho \& Bordons, 2004) with constraints on input, state and output variables at each sampling time, starting from the current state, an open-loop optimal control problem is solved over the defined finite horizon. The first element of the optimal control sequence is applied to the plant. At the next time step, the computation is repeated with new measured variables. Thus, the implementation of the MPC strategy requires a QP solver for the on-line optimization which still requires significant on-line computational effort, which limits MPC applicability.

In our approach the actual output feedback control gain matrix is computed as a convex combination of two gain matrices computed a priori (off-line) : one for constrained and one for unconstrained case such that both gains guarantee performance and robustness properties of closed-loop system. This convex combination is determined by a scalar parameter which is updated on-line in each step. Based on this idea, in the following, the algorithm for constrained control algorithm is developed.

Consider the system (6) where the control $v(t)$ is constrained to evolve in the following set

$$
\Gamma=\left\{v \in R^{m N_{u}}:\left|v_{i}(t)\right| \leq U_{i}, i=1, \ldots m N_{u}\right\}
$$

The aim of this part of chapter is to design the stabilizing output feedback control law for system (6) in the form

$$
v(t)=F C_{f} z(t)
$$

which guarantees that for the initial state $z_{0} \in \Omega(P)=\left\{z(t): z(t)^{T} P z(t) \leq \theta\right\}$ control $v(t)$ belongs to the set (21) for all $t \geq 0$, where $\theta$ is a positive real parameter which determines the size of $\Omega(P)$. Furthermore, $\Omega(P)$ should be such that all $z(t) \in \Omega(P)$ provide $v(t)$ satisfying the relation (21), restricting the values of the control parameters. Moreover, the following ellipsoidal Lyapunov function level set

$$
\Omega(P)=\left\{z(t) \in R^{n N_{2}}: z(t)^{T} P z(t) \leq \theta\right\}
$$

can be proven to be a robust positively invariant region with respect to motion of the closedloop system in the sense of the following definition, (Rohal-Ilkiv, 2004), (Ayd et al., 2008) .

Definition 3. A subset $S_{o} \in R^{\left(n N_{2}\right)}$ is said to be positively invariant with respect to motion of system (6) with control algorithm (22) if for every initial state $z(0)$ inside $S_{0}$ the trajectory $z(t)$ remains in $S_{o}$ for all $t \geq 0$.

Consider that vector $f_{i}$ denotes the i-th row of matrix $F$ and define

$$
L(F)=\left\{z(t) \in R^{\left(n N_{2}\right)}:\left|f_{i} C_{f} z(t)\right| \leq U_{i}, i=1,2 \ldots m N_{u}\right\}
$$


The above set can be rewritten as

$$
L(F)=\left\{z(t) \in R^{\left(n N_{2}\right)}:\left|D_{i} F C_{f} z(t)\right| \leq U_{i}, i=1,2 \ldots m N_{u}\right\}
$$

where $D_{i} \in R^{1 \times m N_{u}}=\{d i j\}, \quad d_{i j}=1, \quad i=j, \quad d_{i j}=0, i \neq j$. The results are summarized in the following theorem.

Theorem 1. The inclusion $\Omega(P) \subseteq L(F)$ is for output feedback control equivalent to

$$
\left[\begin{array}{cc}
P & C^{T} F^{T} D_{i}^{T} \\
D_{i} F C & \lambda_{i}
\end{array}\right] \geq 0, \quad i=1,2, \ldots m N_{u}
$$

where

$$
\lambda_{i} \in<0, \frac{U_{i}^{2}}{\theta}>
$$

Proof. To prove that the inclusion $\Omega(P) \subseteq L(F)$ is equivalent to (25) we use $S$ - procedure in the following way. Rewrite (23) and (24) to the following form

$$
\begin{gathered}
p(z)=z^{T}(t) P z(t)-\theta \leq 0 \\
g_{i}(z)=z^{T}(t) C_{f}^{T} F^{T} D_{i}^{T} D_{i} F C z(t)-U_{i}^{2} \leq 0
\end{gathered}
$$

According to $S-$ procedure the above inclusion is equivalent to the existence of a positive scalar $\lambda_{i}$ such that

$$
g_{i}(z)-\lambda_{i} p(z) \leq 0
$$

or equivalently

$$
z(t)^{T}\left(C_{f}^{T} F^{T} D_{i}^{T} D_{i} F C-\lambda_{i} P\right) z(t)-U_{i}^{2}+\lambda_{i} \theta \leq 0
$$

After some manipulation (26) can be rewritten in the form

$$
\begin{gathered}
{\left[\begin{array}{cc}
C^{T} F^{T} D_{i}^{T} D_{i} F C-\lambda_{i} P & 0 \\
0 & -U_{i}^{2}+\lambda_{i} \theta
\end{array}\right] \leq 0} \\
i=1,2, \ldots m N_{u}
\end{gathered}
$$

The above inequality for block diagonal matrix is equivalent to two inequalities. Using Schur complement formula for the first one the inequality (25) is obtained, which proves the theorem.

In order to check the value of $\theta_{i}$ for $i$ - th input we solve the optimization problem $z(t)^{T} P z(t) \rightarrow$ max, subject to constraints (24), which yields

$$
\theta_{i}=\frac{U_{i}^{2}}{D_{i} F C P^{-1} C^{T} F^{T} D_{i}^{T}}
$$

In the design procedure it should be verified that when parameter $\theta$ decreases the obtained robust positively invariant regions $\Omega(P)$ are nested to region obtained for $\theta+\epsilon, \epsilon>0$.

Assume that we calculate two output feedback gain matrices: $F_{1}$ for unconstrained case and $F_{2}$ for constrained one. Obviously, closed-loop system with the gain matrix $F_{2}$ gives the dynamic behavior slower than the one obtained for $F_{1}$. Consider the output feedback gain matrix $F$ in the form

$$
F=\gamma F_{1}+(1-\gamma) F_{2}, \quad \gamma \in(0,1)
$$


For gain matrices $F_{i}, i=1,2$ we obtain two closed-loop system in the form (13), $A_{c i}=A_{f}+$ $B_{f} F_{i} C_{f}, i=1,2$. Consider the edge between $A_{c 1}$ and $A_{c 2}$, that is

$$
A_{c}=\alpha A_{c 1}+(1-\alpha) A_{c 2}, \quad \alpha \in<0,1>
$$

The following lemma gives the stability conditions for matrix $A_{c}(30)$.

Lemma 2. Consider the stable closed-loop system matrices $A_{c i}, i=1,2$.

- If there exists a positive definite matrix $P_{q}$ such that

$$
A_{c i}^{T} P_{q} A_{c i}-P_{q} \leq 0, \quad i=1,2
$$

then matrix $A_{\mathcal{C}}(30)$ is quadratically stable.

- If there exist two positive definite matrices $P_{1}, P_{2}$ such that they satisfy the parameter dependent quadratic stability conditions, see (Peaucelle et al., 2000), (Grman et al., 2005) the closed-loop system $A_{c}$ is parameter dependent quadratically stable (PDQS).

Remarks

- If closed-loop matrices $A_{c i}, \quad i=1,2$ satisfy (31) the scalar $\gamma$ in (29) may be changed with any rate without violating the closed-loop stability.

- If closed-loop matrices $A_{c i}, \quad i=1,2$ are PDQS, the scalar $\gamma$ in (29) has to be constant but may be unknown.

- The proposed control algorithm (29) is similar to Soft Variable-Structure Control (SVSC), (Adamy \& Fleming, 2004), but in our case, when $\left|v_{i}\right|<<U_{i}$ the feedback gain matrix $F(29)$ gives rather quicker dynamic behavior of the closed-loop system (unconstrained case) than when $\left|v_{i}\right|$ approaches to $U_{i}$.

Algorithm for calculation of $\gamma$ for (29) may be as follows:

$$
\gamma=\min _{i} \frac{U_{i}-\left|v_{i}\right|}{U_{i}}
$$

If accidentally some $\left|v_{i}\right|>U_{i}, \gamma=0$.

The resulting control design procedure is given by the next steps

- Off-line computation stage, compute output feedback gain matrices:

$F_{1}$ for unconstrained case as a solution to (20), where LMI (20) is solved for unknown $P$ and $F$;

$F_{2}$ for constrained case as a solution to (20) and (25).

- On-line computation- in each step:

compute the actual value of scalar parameter $\gamma$, e.g from (32) (where $v_{i}$ is obtained from (12) for $F=F_{1}$;

compute the actual feedback gain matrix from (29) and respective constrained control vector from (12). All on-line computations follow general MPC scheme, i.e. the first part of computed control vector $u(t)$ is applied on real controlled plant and the other part of control vector is used for model prediction. 


\subsection{EXAMPLES}

Two examples are presented to illustrate the qualities of the control design procedure proposed above, namely its ability to cope with robust stability and input constraints without complex computational load. In each example the results of three simulation experiments are compared for closed-loop with output feedback control:

case 1 Unconstrained case for output feedback gain matrix $F_{1}$

case 2 Constrained case for output feedback gain matrix $F_{2}$

case 3 The new proposed control algorithm (29) for output feedback gain matrix $F$.

The input constraint case is studied, in each case maximal value of $u(t)$ is checked; stability is assessed using spectral radius of closed-loop system matrix.

First example serves as a benchmark. The model of double integrator turns to (1) where

$$
\begin{gathered}
A_{o}=\left[\begin{array}{ll}
1 & 0 \\
1 & 1
\end{array}\right] \\
B_{o}=\left[\begin{array}{l}
1 \\
0
\end{array}\right], C=\left[\begin{array}{ll}
0 & 1
\end{array}\right]
\end{gathered}
$$

and uncertainty matrices are

$$
\begin{gathered}
A_{1 u}=\left[\begin{array}{ll}
0.01 & 0.01 \\
0.02 & 0.03
\end{array}\right] \\
B_{1 u}=\left[\begin{array}{c}
0.001 \\
0
\end{array}\right],
\end{gathered}
$$

For the case when number of uncertainty is $p=1$, the number of the respective polytope vertices is $N=2^{p}=2$, the matrices (2) are calculated as follows

$$
A_{1}=A_{o}-A_{1 u}, \quad A_{2}=A_{o}+A_{1 u}, \quad B_{1}=B_{0}-B_{1 u}, \quad B_{2}=B_{o}+B_{1 u}
$$

For the parameters: $\varrho=20000, N_{2}=6, N_{u}=6, Q_{0}=0.1 I, Q_{1}=0.5 I, Q_{2}=\ldots=Q_{6}=I, R=$ $I$, the following results are obtained for unconstrained and constrained cases

- Unconstrained case: Closed - loopmaxeig $=0.8495$. Maximal value of control variable is about $u_{\max }=0.24$.

- Constrained case with $U_{i}=0.1, \theta=1000$, Closed - loopmaxeig $=0.9437$. Maximal value of control variable is about $u_{\max }=0.04$.

Closed-loop step responses for unconstrained and constrained cases are given in Fig.1 and Fig.2, respectively. Closed-loop step responses for the case of in this chapter proposed algorithm are given in Fig.3. Maximal value of control variable is about $u_{\max }=0.08<0.1$.

Input constraints conditions were applied only for plant control variable $u(t)$.

Second example has been borrowed from (Camacho \& Bordons (2004), p.147). The model corresponds to the longitudinal motion of a Boeing 747 airplane. The multivariable process is controlled using a predictive controller based on the output model of the aircraft. Two of the usual command outputs that must be controlled are airspeed that is, velocity with respect to air, and climb rate. Continuous model has been converted to discrete time one with sampling time of 0.1 , the nominal model turns to (1) where

$$
A_{o}=\left[\begin{array}{cccc}
.9996 & .0383 & .0131 & -.0322 \\
-.0056 & .9647 & .7446 & .0001 \\
.002 & -.0097 & .9543 & 0 \\
.0001 & -.0005 & .0978 & 1
\end{array}\right]
$$




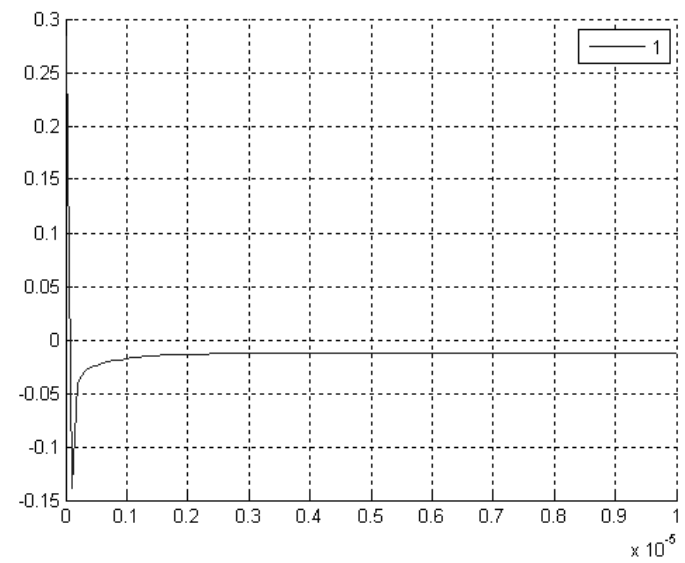

Fig. 1. Dynamic behavior of controlled system for unconstrained case for $u(t)$.

$$
B_{o}=\left[\begin{array}{cc}
.0001 & .1002 \\
-.0615 & .0183 \\
-.1133 & .0586 \\
-.0057 & .0029
\end{array}\right] C=\left[\begin{array}{cccc}
1 & 0 & 0 & 0 \\
0 & -1 & 0 & 7.74
\end{array}\right]
$$

and model uncertainty matrices are

$$
\begin{aligned}
A_{1 u}= & {\left[\begin{array}{cccc}
0 & 0 & 0 & 0 \\
0 & 0.0005 & 0.0017 & 0 \\
0 & 0 & 0.0001 & 0 \\
0 & 0 & 0 & 0
\end{array}\right] } \\
B_{1 u} & =\left[\begin{array}{cc}
0 & 0.12 \\
-0.02 & 0.1 \\
-0.12 & 0 \\
0 & 0
\end{array}\right] 10^{-3}
\end{aligned}
$$

For the case when number of uncertainty is $p=1$, the number of vertices is $N=2^{p}=2$, the matrices (2) are calculated as in example 1. Note that nominal model $A_{0}$ is unstable. Consider $N_{2}=N_{u}=1, \varrho=20000$ and weighting matrices $Q_{0}=Q_{1}=1 I, R_{0}=R_{1}=I$ the following results are obtained:

- Unconstrained case: maximal closed-loop nominal model eigenvalue is Closed -loopmaxeig = 0.9983. Maximal value of control variables are about $u_{1 \max }=9.6, u_{2 \max }=6.3$.

- Constrained case with $U_{i}=1, \theta=40000$ Closed - loopmaxeig $=0.9998$ Maximal values of control variables are about $u_{1 \max }=0.21, u_{2 \max }=0.2$.

Closed-loop nominal model step responses of the above two cases for the input $u(t)$ are given in the Fig.4 and Fig.5, respectively. Closed-loop step responses for in the paper proposed control algorithm (29) and (32) are in Fig.6. Maximal values of control variables are about $u_{1 \max }=0.75<1, u_{2 \max }=0.6<1$. Input constraint conditions were applied only for plant control variable $u(t)$. Both examples show that using tuning parameter $\theta$ the demanded input 


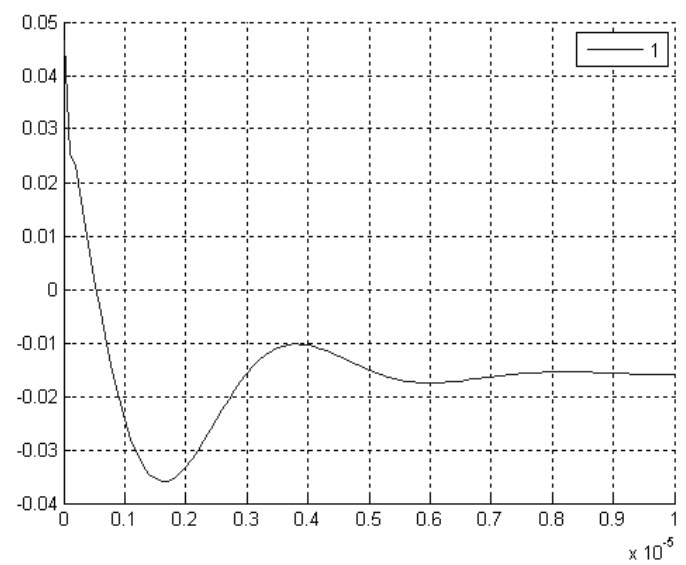

Fig. 2. Dynamic behavior of controlled system for constrained case for $u(t)$.

constraints can be reached with high accuracy. The initial guess of $\theta$ can be obtained from (28). It can be seen that the proposed control scheme provides reasonable results : the response in case 3 (Fig.3 , Fig. 6) are quicker than those in case 2 (Fig.2, Fig.5), while the computation load has not much increased comparing to case 2 .

\section{ROBUST MPC DESIGN: SEQUENTIAL APPROACH}

\subsection{INTRODUCTION}

In this part a new MPC algorithm is proposed pursuing the idea of (Veselý \& Rosinová, 2009). The proposed robust MPC control algorithm is designed sequentially. The respective sequential robust MPC design procedure consists from two steps. In the first step and one step ahead prediction horizon, the necessary and sufficient robust stability conditions have been developed for MPC and polytopic system with output feedback, using generalized parameter dependent Lyapunov matrix $P(\alpha)$. The proposed robust MPC algorithm ensures parameter dependent quadratic stability (PDQS) and guaranteed cost. In the second step of design procedure, the nominal plant model is used to design the predicted input variables $u(t+1), \ldots u\left(t+N_{2}-1\right)$ so that the robust closed-loop stability of MPC and guaranteed cost are ensured. Thus, input variable $u(t)$ guarantees the performance and robustness properties of closed-loop system and predicted input variables $u(t+1), \ldots u\left(t+N_{2}-1\right)$ guarantee the performance and closed-loop stability of uncertain plant model and nominal model prediction. Note that within sequentially design procedure the degree of plant model does not change when the output prediction horizon changes.

This part of chapter is organized as follows: Section 2.2 presents preliminaries and problem formulation. In Section 2.3 the main results are given and finally, in Section 2.4 two examples solved using Yalmip BMI solvers show the effectiveness of the proposed method. 


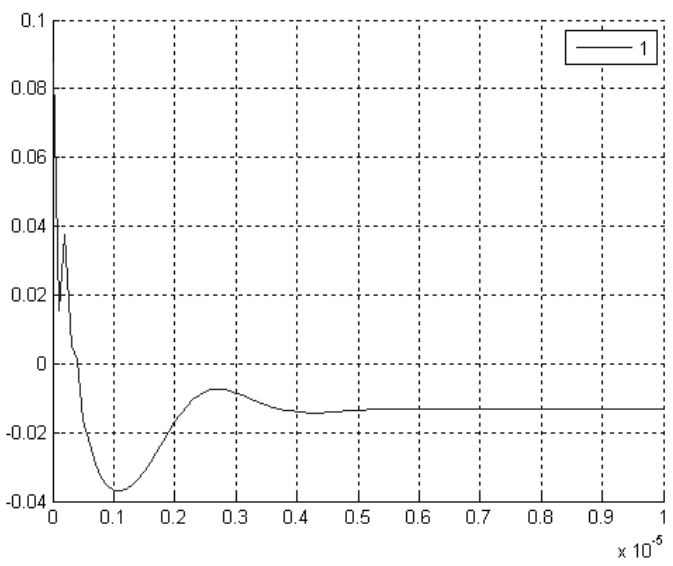

Fig. 3. Dynamic behavior of controlled system with the proposed algorithm for $u(t)$.

\subsection{PROBLEM FORMULATION AND PRELIMINARIES}

For readers convenience, uncertain plant model and respective preliminaries are briefly recalled. A time invariant linear discrete-time uncertain polytopic system is

$$
\begin{gathered}
x(t+1)=A(\alpha) x(t)+B(\alpha) u(t) \\
y(t)=C x(t)
\end{gathered}
$$

where $x(t) \in R^{n}, u(t) \in R^{m}, y(t) \in R^{l}$ are state, control and output variables of the system, respectively; $A(\alpha), B(\alpha)$ belong to the convex set

$$
\begin{gathered}
S=\left\{A(\alpha) \in R^{n \times n}, B(\alpha) \in R^{n \times m}\right\} \\
\left\{A(\alpha)=\sum_{j=1}^{N} A_{j} \alpha_{j} \quad B(\alpha)=\sum_{j=1}^{N} B_{j} \alpha_{j}, \alpha_{j} \geq 0\right\}, j=1,2 \ldots N, \sum_{j=1}^{N} \alpha_{j}=1
\end{gathered}
$$

Matrix $C$ is constant known matrix of corresponding dimension. Jointly with the system (33), the following nominal plant model will be used.

$$
\begin{gathered}
x(t+1)=A_{o} x(t)+B_{o} u(t) \\
y(t)=C x(t)
\end{gathered}
$$

where $\left(A_{o}, B_{0}\right) \in S$ are any matrices with constant entries. The problem studied in this part of chapter can be summarized as follows: in the first step, parameter dependent quadratic stability conditions for output feedback and one step ahead robust model predictive control are derived for the polytopic system (33), (34), when control algorithm is given as

$$
u(t)=F_{11} y(t)+F_{12} y(t+1)
$$

and in the second step of design procedure, considering a nominal model (35) and a given prediction horizon $\mathrm{N}_{2}$ a model predictive control is designed in the form:

$$
u(t+k-1)=F_{k k} y(t+k-1)+F_{k k+1} y(t+k)
$$




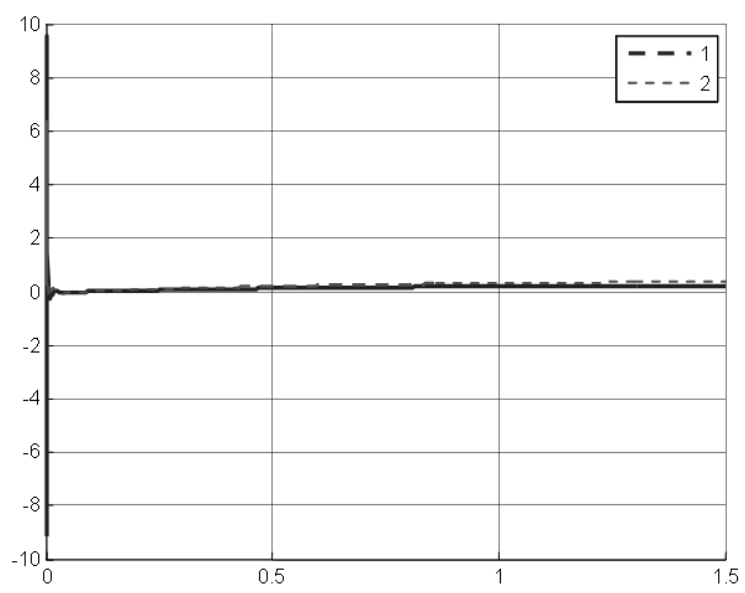

Fig. 4. Dynamic behavior of unconstrained controlled system for $u(t)$.

where $F_{k i} \in R^{m \times l}, k=2,3, \ldots N_{2} ; i=k+1$ are output (state) feedback gain matrices to be determined so that cost function given below is optimal with respect to system variables. We would like to stress that $y(t+k-1), y(t+1)$ are predicted outputs obtained from predictive model (44).

Substituting control algorithm (36) to (33) we obtain

$$
x(t+1)=D_{1}(j) x(t)
$$

where

$$
\begin{gathered}
D_{1}(j)=A_{j}+B_{j} K_{1}(j) \\
K_{1}(j)=\left(I-F_{12} C B_{j}\right)^{-1}\left(F_{11} C+F_{12} C A_{j}\right), j=1,2, \ldots N
\end{gathered}
$$

For the first step of design procedure, the cost function to be minimized is given as

$$
J_{1}=\sum_{t=0}^{\infty} J_{1}(t)
$$

where

$$
J_{1}(t)=x(t)^{T} Q_{1} x(t)+u(t)^{T} R_{1} u(t)
$$

and $Q_{1}, R_{1}$ are positive definite matrices of corresponding dimensions. For the case of $k=2$ we obtain

$$
u(t+1)=F_{22} C D_{1}(j) x(t)+F_{23} C\left(A_{o} D_{1}(j) x(t)+B_{o} u(t+1)\right)
$$

or

and closed-loop system

$$
u(t+1)=K_{2}(j) x(t)
$$

$$
x(t+2)=\left(A_{o} D_{1}(j)+B_{o} K_{2}(j)\right) x(t)=D_{2}(j) x(t), j=1,2, \ldots N
$$




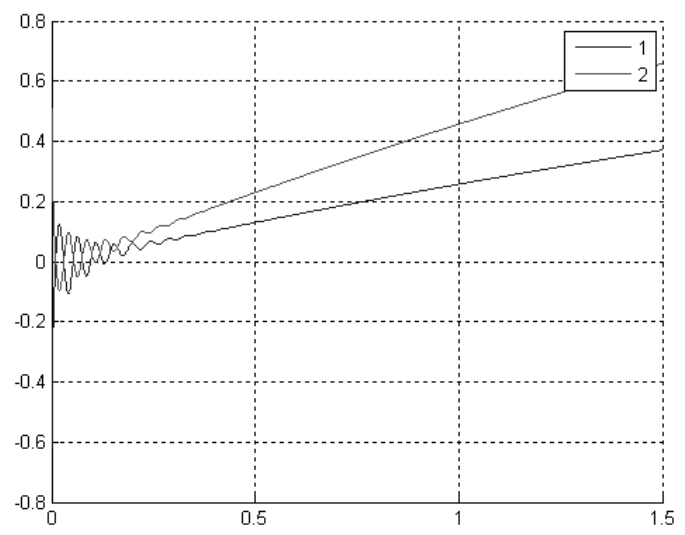

Fig. 5. Dynamic behavior of constrained controlled system for $u(t)$.

Sequentially, for $k=N_{2} \geq 2$ step prediction, we obtain the following closed-loop system

$$
x(t+k)=\left(A_{o} D_{k-1}(j)+B_{o} K_{k}(j)\right) x(t)=D_{k}(j) x(t)
$$

where

$$
\begin{aligned}
D_{0}=I, D_{k}(j) & =A_{o} D_{k-1}(j)+B_{o} K_{k}(j) \quad k=2,3, \ldots, N_{2} ; \quad j=1,2, \ldots N \\
K_{k}(j) & =\left(I-F_{k k+1} C B_{o}\right)^{-1}\left(F_{k k} C+F_{k k+1} C A_{o}\right) D_{k-1}(j)
\end{aligned}
$$

For the second step of robust MPC design procedure and $k$ prediction horizon the cost function to be minimized is given as

$$
J_{k}=\sum_{t=0}^{\infty} J_{k}(t)
$$

where

$$
J_{k}(t)=x(t)^{T} Q_{k} x(t)+u(t+k-1)^{T} R_{k} u(t+k-1)
$$

and $Q_{k}, R_{k}, k=2,3, \ldots N_{2}$ are positive definite matrices of corresponding dimensions. We proceed with two corollaries following from Definition 2 and Lemma 1.

Corollary 1

The closed-loop system matrix of discrete-time system (1) is robustly stable if and only if there exists a symmetric positive definite parameter dependent Lyapunov matrix $0<P(\alpha)=$ $P(\alpha)^{T}<I \varrho$ such that

$$
-P(\alpha)+D_{1}(\alpha)^{T} P(\alpha) D_{1}(\alpha) \leq 0
$$

where $D_{1}(\alpha)$ is the closed-loop polytopic system matrix for system (33). The necessary and sufficient robust stability condition for closed-loop polytopic system with guaranteed cost is given by the recent result (Rosinová et al., 2003).

Corollary 2

Consider the system (33) with control algorithm (36). Control algorithm (36) is the guaranteed cost control law for the closed-loop system if and only if the following condition holds

$$
B_{e}=D_{1}(\alpha)^{T} P(\alpha) D_{1}(\alpha)-P(\alpha)+Q_{1}+\left(F_{11} C+F_{12} C D_{1}(\alpha)\right)^{T} R_{1}\left(F_{11} C+\right.
$$




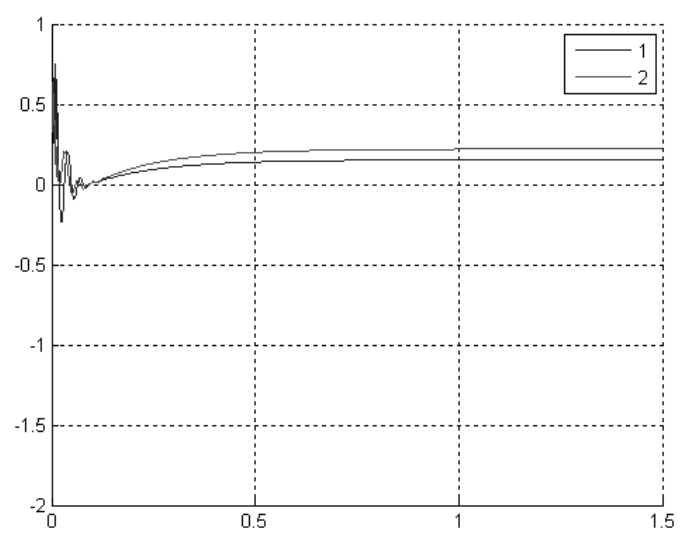

Fig. 6. Dynamic behavior for proposed control algorithm (29) and (32) for $u(t)$.

$$
\left.+F_{12} C D_{1}(\alpha)\right) \leq 0
$$

For the nominal model and $k=1,2, \ldots N_{2}$ the model prediction can be obtained in the form

$$
\begin{gathered}
z(t+1)=A_{f} z(t)+B_{f} v(t) \\
y_{f}(t)=C_{f} z(t)
\end{gathered}
$$

where

$$
\begin{gathered}
z(t)^{T}=\left[x(t)^{T} \ldots x\left(t+N_{2}-1\right)^{T}\right] \\
v(t)^{T}=\left[u(t)^{T} \ldots u\left(t+N_{2}-1\right)^{T}\right] \\
A_{f}(t)^{T}=\left[y(t)^{T} \ldots y\left(t+N_{2}-1\right)^{T}\right] \\
{\left[\begin{array}{ccccc}
A_{o} & 0 & 0 & \ldots & 0 \\
A_{o} D_{1} & 0 & 0 & \ldots & 0 \\
A_{o} D_{2} & 0 & 0 & \ldots & 0 \\
\ldots & \ldots & \ldots & \ldots & \ldots \\
A_{0} D_{N_{2}-1} & 0 & 0 & \ldots & 0
\end{array}\right] \in R^{n N_{2} \times n N_{2}}} \\
B_{f}=\text { blockdiag }\left\{B_{o}\right\}_{n N_{2} \times m N_{2}} \\
C_{f}=\text { blockdiag }\{C\}_{l N_{2} \times n N_{2}}
\end{gathered}
$$

Remarks

- Control algorithm for $k=N_{2}$ is $u\left(t+N_{2}-1\right)=F_{N_{2} N_{2}} y\left(t+N_{2}-1\right)$.

- If one wants to use control horizon $N_{u}<N_{2}$ (Camacho \& Bordons, 2004), the control algorithm is $u(t+k-1)=0, K_{k}=0, F_{N_{u+1}} N_{u+1}=0, F_{N_{u+1} N_{u+2}}=0$ for $k>N_{u}$.

- Note that model prediction (44) is calculated using nominal model (35), that is $D_{0}=$ $I, D_{k}=A_{o} D_{k-1}+B_{o} K_{k}, D_{k}(j)$ is used robust controller design procedure. 


\subsection{MAIN RESULTS}

\subsubsection{Robust MPC controller design. First step}

The main results for the first step of design procedure can be summarized in the following theorem.

Theorem 2.

The system (33) with control algorithm (36) is parameter dependent quadratically stable with parameter dependent Lyapunov function $V(t)=x(t)^{T} P(\alpha) x(t)$ if and only if there exist matrices $N_{11}, N_{12}, F_{11}, F_{12}$ such that the following bilinear matrix inequality holds.

$$
B_{e}=\left[\begin{array}{ll}
G_{11} & G_{12} \\
G_{12}^{T} & G_{22}
\end{array}\right] \leq 0
$$

where

$$
\begin{gathered}
G_{22}=N_{12}^{T} A_{\mathcal{C}}(\alpha)+A_{\mathcal{C}}(\alpha)^{T} N_{12}-P(\alpha)+Q_{1}+C^{T} F_{11}^{T} R_{1} F_{11} C \\
G_{12}^{T}=A_{\mathcal{C}}(\alpha)^{T} N_{11}+N_{12}^{T} M_{\mathcal{C}}(\alpha)+C^{T} F_{11}^{T} R_{1} F_{12} C \\
G_{11}=N_{22}^{T} M_{\mathcal{c}}(\alpha)+M_{\mathcal{c}}(\alpha)^{T} N_{22}+C^{T} F_{12}^{T} R_{1} F_{12} C+P(\alpha) \\
M_{\mathcal{c}}(\alpha)=B(\alpha) F_{12} C-I \\
A_{\mathcal{c}}(\alpha)=A(\alpha)+B(\alpha) F_{11} C
\end{gathered}
$$

Note that (45) is affine with respect to $\alpha$. Substituting (34) and $P(\alpha)=\sum_{i=1}^{N} \alpha_{i} P_{i}$ to (45) the following BMI is obtained for the polytopic system

$$
B_{i e}=\left[\begin{array}{ll}
G_{11 i} & G_{12 i} \\
G_{12 i}^{T} & G_{22 i}
\end{array}\right] \leq 0 \quad i=1,2, \ldots N
$$

where

$$
\begin{gathered}
G_{11 i}=N_{22}^{T} M_{c i}+M_{c i}^{T} N_{22}+C^{T} F_{12}^{T} R_{1} F_{12} C+P_{i} \\
G_{12 i}^{T}=A_{c i}^{T} N_{22}+N_{12}^{T} M_{c i}+C^{T} F_{11}^{T} R_{1} F_{12} C \\
G_{22 i}=N_{12}^{T} A_{c i}+A_{c i}^{T} N_{12}-P_{i}+Q_{1}+C^{T} F_{11}^{T} R_{1} F_{11} C \\
M_{c i}=B_{i} F_{12} C-I \quad A_{c i}=A_{i}+B_{i} F_{11} C
\end{gathered}
$$

Proof. For the proof of this theorem see the proof of Theorem 3 .

If the solution of (46) is feasible with respect to symmetric matrices $P_{i}=P_{i}^{T}>0, i=1,2 \ldots N$, and matrices $N_{11}, N_{12}$, within the convex set defined by (34), the gain matrices $F_{11}, F_{12}$ ensure the guaranteed cost and parameter dependent quadratic stability (PDQS) of closed-loop polytopic system for one step ahead predictive control.

Note that:

- For concrete matrix $P(\alpha)=\sum_{i=1}^{N} \alpha_{i} P_{i}$ BMI robust stability conditions "if and only if" in (45) reduces in (46) to BMI conditions " if".

- If in (46) $P_{i}=P_{j}=P, i \neq j=1,2 \ldots N$, the feasible solution of (46) with respect to matrices $N_{11}, N_{12}$, and symmetric positive definite matrix $P$ gives the gain matrices $F_{11}, F_{12}$ guaranteeing quadratic stability and guaranteed cost for one step ahead predictive control for the closed-loop polytopic system within the convex set defined by (34). Quadratic stability gives more conservative results than PDQS. Conservatism of real results depend on the concrete examples.

Assume that the BMI solution of (46) is feasible, then for nominal plant one can calculate matrices $D_{1}$ and $K_{1}$ using (38). For the second step of MPC design procedure, the obtained nominal model will be used. 


\subsubsection{Model predictive controller design. Second step}

The aim of the second step of predictive control design procedure is to design gain matrices $F_{k k}, F_{k k+1}, k=2,3, \ldots N_{2}$ such that the closed-loop system with nominal model is stable with guaranteed cost. In order to design model predictive controller with output feedback in the second step of design procedure we proceed with the following corollary and theorem.

Corollary 3

The closed-loop system (40) is stable with guaranteed cost iff the following inequality holds

$$
B_{e k}(t)=\Delta V_{k}(t)+x(t)^{T} Q_{k} x(t)+u(t+k-1)^{T} R_{k} u(t+k-1) \leq 0
$$

where $\Delta V_{k}(t)=V_{k}(t+k)-V_{k}(t)$ and $V_{k}(t)=x(t)^{T} P_{k} x(t), P_{k}=P_{k}^{T}>0, k=2,3, \ldots N_{2}$.

Theorem 3

The closed-loop system (40) is robustly stable with guaranteed cost iff for $k=2,3, \ldots N_{2}$ there exist matrices

$$
F_{k k}, F_{k k+1}, N_{k 1} \in R^{n \times n}, N_{k 2} \in R^{n \times n}
$$

and positive definite matrix $P_{k}=P_{k}^{T} \in R^{n \times n}$ such that the following bilinear matrix inequality holds

$$
B_{e 2}=\left[\begin{array}{ll}
G_{k 11} & G_{k 12} \\
G_{k 12}^{T} & G_{k 22}
\end{array}\right] \leq 0
$$

where

$$
\begin{gathered}
G_{k 11}=N_{k 1}^{T} M_{c k}+M_{c k}^{T} N_{k 1}+C^{T} F_{k k+1}^{T} R_{k} F_{k k+1} C+P_{k} \\
G_{k 12}^{T}=D_{k-1}(j)^{T} C^{T} F_{k k}^{T} R_{k} F_{k k+1} C+D_{k-1}(j)^{T} A_{c k}^{T} N_{k 1}+N_{k 2}^{T} M_{c k} \\
G_{k 22}=Q_{k}-P_{k}+D_{k-1}(j)^{T} C^{T} F_{k k}^{T} R_{k} F_{k k} C D_{k-1}(j) \\
+N_{k 2}^{T} A_{c k} D_{k-1}(j)+D_{k-1}(j)^{T} A_{c k}^{T} N_{k 2}
\end{gathered}
$$

and

$$
\begin{gathered}
M_{c k}=B_{0} F_{k k+1} C-I ; \quad A_{c k}=A_{0}+B_{0} F_{k k} C \\
D_{k}(j)=A_{0} D_{k-1}(j)+B_{0} K_{k}(j) \\
K_{k}(j)=\left(I-F_{k k+1} C B_{0}\right)^{-1}\left(F_{k k} C+F_{k k+1} C A_{0}\right) D_{k-1}(j), \quad j=1,2, \ldots N
\end{gathered}
$$

Proof. Sufficiency.

The closed-loop system (40) can be rewritten as follows

$$
x(t+k)=-\left(M_{c k}\right)^{-1} A_{c k} D_{k-1}(j) x(t)=A_{c l k} x(t)
$$

Since the matrix ( $j$ is omitted)

$$
U_{k}^{T}=\left[-D_{k-1}^{T} A_{c k}^{T}\left(M_{c k}\right)^{-1} \quad I\right]
$$

has full row rank, multiplying (48) from left and right side the inequality equivalent to (47) is obtained. Multiplying the results from left by $x(t)^{T}$ and right by $x(t)$, taking into account the closed-loop matrix (49), the inequality (47) is obtained, which proves the sufficiency.

Necessity.

Suppose that for k-step ahead model predictive control there exists such matrix $0<P_{k}=$ 
$P_{k}^{T}<I \rho$ that (48) holds. Necessarily, there exists a scalar $\beta>0$ such that for the first difference of Lyapunov function in (47) holds

$$
A_{c l k}^{T} P_{k} A_{c l k}-P_{k} \leq-\beta\left(A_{c l k}^{T} A_{c l k}\right)
$$

The inequality (50) can be rewritten as

$$
A_{c l k}^{T}\left(P_{k}+\beta I\right) A_{c l k}-P_{k} \leq 0
$$

Using Schur complement formula we obtain

$$
\left[\begin{array}{cc}
-P_{k} & -A_{c l k}^{T}\left(P_{k}+\beta I\right) \\
\left(P_{k}+\beta I\right) A_{c l k} & -\left(P_{k}+\beta I\right)
\end{array}\right] \leq 0
$$

taking

one obtains

$$
\begin{gathered}
N_{k 1}=-\left(M_{c k}\right)^{-1}\left(P_{k}+\beta I / 2\right) \\
N_{k 2}^{T}=-D_{k-1}^{T} A_{c k}^{T}\left(M_{c k}^{-1}\right)^{T} M_{c k}^{-1} \beta / 2
\end{gathered}
$$

$$
\begin{gathered}
-A_{c l k}^{T}\left(P_{k}+\beta I\right)=D_{k-1}^{T} A_{c k}^{T} N_{k 1}+N_{k 2}^{T} M_{c k} \\
-P_{k}=-P_{k}+N_{k 2}^{T} A_{c k} D_{k-1}+D_{k-1}^{T} \\
A_{c k}^{T} N_{k 2}+\beta\left(D_{k-1}^{T} A_{c k}^{T}\left(M_{c k}^{-1}\right)^{T} M_{c k}^{-1} A_{c k} D_{k-1}\right) \\
-\left(P_{k}+\beta I\right)=2 M_{c k} N_{k 1}+P_{k}
\end{gathered}
$$

Substituting (52) to (51) for $\beta \rightarrow 0$ the inequality (48) is obtained for the case of $Q_{k}=0, R_{k}=0$. If one substitutes to the second part of (47) for $u(t+k-1)$ from (37), rewrites the obtained result to matrix form and takes sum of it with the above matrix, inequality (48) is obtained, which proves the necessity. It completes the proof.

If there exists a feasible solution of (48) with respect to matrices $F_{k k}, F_{k k+1}, N_{k 1} \in R^{n \times n}, N_{k 2} \in$ $R^{n \times n}, k=2,3, \ldots N_{2}$ and positive definite matrix $P_{k}=P_{k}^{T} \in R^{n \times n}$, then the designed MPC ensures quadratic stability of the closed-loop system and guaranteed cost.

\section{Remarks}

- Due to the proposed design philosophy, predictive control algorithm $u(t+k), k \geq 1$ is the function of corresponding performance term (39) and previous closed-loop system matrix.

- In the proposed design approach constraints on system variables are easy to be implemented by LMI using a notion of invariant set (Ayd et al., 2008), (Rohal-Ilkiv, 2004) (see Section 1.3).

- The proposed MPC with sequential design is a special case of classical MPC. Sequential MPC may not provide "better" dynamic behavior than classical one but it is another approach to the design of MPC.

- Note that in the proposed MPC sequential design procedure, the size of system does not change when $\mathrm{N}_{2}$ increases.

- If there exists feasible solution for both steps in the convex set (34), the proposed control algorithm (37) guarantees the PDQS and robustness properties of closed-loop MPC system with guaranteed cost. 
The sequential robust MPC design procedure can be summarized in the following steps:

- Design of robust MPC controller with control algorithm (36) by solving (46).

- Calculate matrices $K_{1}, D_{1}$ and $K_{1}(j), D_{1}(j), j=1,2, \ldots N$ given in (38) for nominal and uncertain model of system.

- For a given $k=2,3, \ldots N_{2}$ and control algorithm (37), sequentially calculate $F_{k k}, F_{k k+1}$ by solving (48) with $K_{k}, D_{k}$ given in (40).

- Calculate matrices $A_{f}, B_{f}, C_{f}(44)$ for model prediction.

\subsection{EXAMPLES}

Example 1. First example is the same as in section 1.5, it serves as a benchmark. The model of double integrator turns to (35) where

$$
\begin{gathered}
A_{o}=\left[\begin{array}{ll}
1 & 0 \\
1 & 1
\end{array}\right] \\
B_{o}=\left[\begin{array}{l}
1 \\
0
\end{array}\right], C=\left[\begin{array}{ll}
0 & 1
\end{array}\right]
\end{gathered}
$$

and uncertainty matrices are

$$
\begin{gathered}
A_{1 u}=\left[\begin{array}{ll}
0.01 & 0.01 \\
0.02 & 0.03
\end{array}\right] \\
B_{1 u}=\left[\begin{array}{c}
0.001 \\
0
\end{array}\right],
\end{gathered}
$$

For the case when number of uncertainties $p=1$, the number of vertices is $N=2^{p}=2$, the matrices (34) are calculated as

$$
\begin{gathered}
A_{1}=A_{n}-A_{1 u}, \quad A_{2}=A_{n}+A_{1 u} \\
B_{1}=B_{n}-B_{1 u}, \quad B_{2}=B_{n}+B_{1 u}
\end{gathered}
$$

For the parameters: $\varrho=20000$, prediction and control horizons $N_{2}=4, N_{u}=4$, performance matrices $R_{1}=\ldots R_{4}=1, Q_{1}=.1 I, Q_{2}=.5 I, Q_{3}=I, Q_{4}=5 I$, the following results are obtained using the sequential design approach proposed in this part :

- For prediction $k=1$, the robust control algorithm is given as

$$
u(t)=F_{11} y(t)+F_{12} y(t+1)
$$

From (46), one obtains the gain matrices $F_{11}=0.9189 ; F_{12}=-1.4149$. The eigenvalues of closed-loop first vertex system model are as follows

$$
\text { Eig }(\text { Closed }- \text { loop })=\{0.2977 \pm 0.0644 i\}
$$

- For $k=2$, control algorithm is

$$
u(t+1)=F_{22} y(t+1)+F_{23} y(t+2)
$$

In the second step of design procedure control gain matrices obtained solving (48) are $F_{22}=0.4145 ; F_{23}=-0.323$. The eigenvalues of closed-loop first vertex system model are

$$
\operatorname{Eig}(\text { Closed }- \text { loop })=\{0.1822 \pm 0.1263 i\}
$$


- For $\mathrm{k}=3$, control algorithm is

$$
u(t+2)=F_{33} y(t+2)+F_{34} y(t+3)
$$

In the second step of design procedure the obtained control gain matrices are $F_{33}=$ $0.2563 ; F_{34}=-0.13023$. The eigenvalues of closed-loop first vertex system model are

$$
\operatorname{Eig}(\text { Closed }- \text { loop })=\{0.1482 \pm 0.051 i\}
$$

- For prediction $k=N_{2}=4$, control algorithm is

$$
u(t+3)=F_{44} y(t+3)+F_{45} y(t+4)
$$

In the second step the obtained control gain matrices are $F_{44}=0.5797 ; F_{45}=0.0$. The eigenvalues of closed-loop first vertex model system are

$$
\operatorname{Eig}(\text { Closed }- \text { loop })=\{0.1002 \pm 0.145 i\}
$$

Example 2. Nominal model for the second example is

$$
\begin{gathered}
A_{O}=\left[\begin{array}{ccccc}
0.6 & 0.0097 & 0.0143 & 0 & 0 \\
0.012 & 0.9754 & 0.0049 & 0 & 0 \\
-0.0047 & 0.01 & 0.46 & 0 & 0 \\
0.0488 & 0.0002 & 0.0004 & 1 & 0 \\
-0.0001 & 0.0003 & 0.0488 & 0 & 1
\end{array}\right] \\
B_{0}=\left[\begin{array}{cc}
0.0425 & 0.0053 \\
0.0052 & 0.01 \\
0.0024 & 0.0001 \\
0 & 0.0012
\end{array}\right] C=\left[\begin{array}{lllll}
1 & 0 & 0 & 0 & 0 \\
0 & 0 & 1 & 0 & 0 \\
0 & 0 & 0 & 1 & 0 \\
0 & 0 & 0 & 0 & 1
\end{array}\right]
\end{gathered}
$$

The linear affine type model of uncertain system (34) is in the form

$$
\begin{gathered}
A_{i}=A_{n}+\theta_{1} A_{1 u} ; \quad B_{i}=B_{n}+\theta_{1} B_{1 u} \\
C_{i}=C, i=1,2
\end{gathered}
$$

where $A_{1 u}, B_{1 u}$ are uncertainty matrices with constant entries, $\theta_{1}$ is an uncertain real parameter $\theta_{1} \in\left\langle\theta_{1}, \hat{\theta}_{1}\right\rangle$. When lower and upper bounds of uncertain parameter $\theta_{1}$ are substituted to the affine type model, the polytopic system (33) is obtained. Let $\theta_{1} \in<-1,1>$ and

$$
\begin{gathered}
A_{1 u}=\left[\begin{array}{ccccc}
0.025 & 0 & 0 & 0 & 0 \\
0 & 0.021 & 0 & 0 & 0 \\
0 & 0 & 0.0002 & 0 & 0 \\
0.001 & 0 & 0 & 0 & 0 \\
0 & 0 & 0.0001 & 0 & 0
\end{array}\right] \\
B_{1 u}=\left[\begin{array}{cc}
0.0001 & 0 \\
0 & 0.001 \\
0 & 0.0021 \\
0 & 0 \\
0 & 0
\end{array}\right]
\end{gathered}
$$


In this example two vertices $(N=2)$ are calculated. The design problem is: Design two PS(PI) model predictive robust decentralized controllers for plant input $u(t)$ and prediction horizon $N_{2}=5$ using sequential design approach. The cost function is given by the following matrices

$$
\begin{gathered}
Q_{1}=Q_{2}=Q_{3}=I, R_{1}=R_{2}=R_{3}=I, \\
Q_{4}=Q_{5}=0.5 I, R_{4}=R_{5}=I
\end{gathered}
$$

In the first step, calculation for the uncertain system (33) yields the robust control algorithm

$$
u(t)=F_{11} y(t)+F_{12} y(t+1)
$$

where matrix $F_{11}$ with decentralized output feedback structure containing two PS controllers, is designed. From (46), the gain matrices $F_{11}, F_{12}$ are obtained

$$
F_{11}=\left[\begin{array}{cccc}
-18.7306 & 0 & -42.4369 & 0 \\
0 & 8.8456 & 0 & 48.287
\end{array}\right]
$$

where decentralized proportional and integral gains for the first controller are

$$
K_{1 p}=18.7306, K_{1 i}=42.4369
$$

and for the second one

$$
K_{2 p}=-8.8456, K_{2 i}=-48.287
$$

Note that in $F_{11}$ sign - shows the negative feedback. Because predicted output $y(t+1)$ is obtained from prediction model (44), for output feedback gain matrix $F_{12}$ there is no need to use decentralized control structure

$$
F_{12}=\left[\begin{array}{cccc}
-22.0944 & 20.2891 & -10.1899 & 18.2789 \\
-29.3567 & 8.5697 & -28.7374 & -40.0299
\end{array}\right]
$$

In the second step of design procedure, using (48) for nominal model, the matrices (37) $F_{k k}, F_{k k+1}, k=$ $2,3,4,5$ are calculated. The eigenvalues of closed-loop first vertex system model for $\mathrm{N}_{2}=$ $N_{u}=5$ are

$$
\text { Eig }(\text { Closed }- \text { loop })=\{-0.0009 ;-0.0087 ; 0.9789 ; 0.8815 ; 0.8925\}
$$

Feasible solutions of bilinear matrix inequality have been obtained by YALMIP with PENBMI solver.

\section{CONCLUSION}

The first part of chapter addresses the problem of designing the output/state feedback robust model predictive controller with input constraints for output and control prediction horizons $N_{2}$ and $N_{u}$. The main contribution of the presented results is twofold: The obtained robust control algorithm guarantees the closed-loop system quadratic stability and guaranteed cost under input constraints in the whole uncertainty domain. The required on-line computation load is significantly less than in MPC literature (according to the best knowledge of authors), which opens possibility to use this control design scheme not only for plants with slow dynamics but also for faster ones. At each sample time the calculation of proposed control algorithm reduces to a solution of simple equation. Finally, two examples illustrate the effectiveness of the proposed method. The second part of chapter studies the problem of design 
a new MPC with special control algorithm. The proposed robust MPC control algorithm is designed sequentially, the degree of plant model does not change when the output prediction horizon changes. The proposed sequential robust MPC design procedure consists of two steps: In the first step for one step ahead prediction horizon the necessary and sufficient robust stability conditions have been developed for MPC and the polytopic system with output feedback, using generalized parameter dependent Lyapunov matrix $P(\alpha)$. The proposed robust MPC ensures parameter dependent quadratic stability (PDQS) and guaranteed cost. In the second step of design procedure the uncertain plant and nominal model with sequential design approach is used to design the predicted input variables $u(t+1), \ldots u\left(t+N_{2}-1\right)$ so that to ensure the robust closed-loop stability of MPC with guaranteed cost. Main advantages of the proposed sequential method are that the design plant model degree is independent on prediction horizon $\mathrm{N}_{2}$; robust controller design procedure ensures PDQS and guaranteed cost and the obtained results are easy to be implemented in real plant. In the proposed design approach, constraints on system variables are easy to be implemented by LMI (BMI) using a notion of invariant set. Feasible solution of BMI has been obtained by Yalmip with PENBMI solver.

\section{ACKNOWLEDGMENT}

The work has been supported by Grant N 1/0544/09 of the Slovak Scientific Grant Agency.

\section{References}

Adamy, J. \& Flemming, A. ( 2004) Soft variable-structure controls: a survey, Automatica, 40, 1821-1844.

Ayd, H., Mesquine, F. \& Aitrami, M. (2008) Robust control for uncertain linear systems with state and control constraints. In:Proc. of the 17th World Congress IFAC, Seoul, Korea, 2008, 1153-1158.

Bouzouita, B., Bouani, F. \& Ksouri, M. (2007) Efficient Implementation of Multivariable MPC with Parametric Uncertainties, In:Proc. ECC 2007, Kos, Greece, TuB12.4, CD-ROM.

Camacho, E.F \& Bordons, C. (2004)Model predictive control, Springer-Verlag London Limited.

Casavola, A., Famularo, D. \& Franze, G. (2004) Robust constrained predictive control of uncertain norm-bounded linear systems. Automatica, 40, 1865-1776.

Clarke, D.W. \& Mohtadi, C. (1989) Properties of generalized predictive control. Automatica, 25(6), 859-875.

Clarke, D.W. \& Scattolini, R.(1991) Constrained Receding-horizon Predictive Control. Proceedings IEE 138,(4), 347-354.

Dermicioglu, H. \& Clarke, D.W. (1993) Generalized predictive control with end-point weighting. IEE Proc , 140, Part D(4): 275-282, 1993.

Ding, B., Xi, Y., Cychowski, M.T. \& O'Mahony, T. (2008) A synthesis approach for output robust constrained model predictive control, Automatica,44, 258-264.

Ebihara, Y., Peaucelle, D., Arzelier, D. \& Hagivara, T. (2006) Robust $H_{2}$ Performance Analysis of Uncertain LTI Systems via Polynomially Parameter Dependent Lyapunov functions. In:Preprint of the 5th IFAC Symposium on Robust Control Design, ROCOND 06, Toulouse, France, July 5-7, 2006 CD-ROM.

Grman, L., Rosinová, D., Veselý, V. \& Kozáková, A. (2005) Robust stability conditions for polytopic systems. Int. Journal of Systems Science, Vol36, N15, 961-973. 
Janík, M., Miklovicová, E. \& Mrosko, M. (2008) Predictive control of nonlinear systems. ICIC Express Letters, Vol. 2, N3, 239-244.

Kothare, M.V., Balakrishnan, V, \& Morari, M. (1996) Robust Constrained Model Predictive Control using Linear Matrix Inequalities, Automatica , Vol 32, N10, 1361-1379.

Krokavec, D. \& Filasová, A. (2003) Quadratically stabilized discrete-time robust LQ control. In:Control System Design , Proc. of the 2nd IFAC Conf., Bratislava, 375-380.

Kuwata, Y., Richards, A. \& How, J. (2007) Robust Receding Horizon using Generalized Constraint Tightening, In:Proc. ACC, New Yourk, CD-ROM.

Lovas, Ch. , Seron, M.M. \& Goodwin, G.C. (2007) Robust Model Predictive Control of Input-Constrained Stable Systems with Unstructured Uncertainty, In:Proc. ECC, Kos, Greece, CD-ROM.

Maciejovski, J.M. (2002) Predictive Control with Constraints. Prentice Hall

Mayne, D.Q., Rawlings, J.B.,Rao, C.V. \& Scokaert, P.O.M. (2000) Contrained model predictive control: stability and optimality. Automatica 36: 789-814.

de Oliveira, M.C., Camino, J.F. \& Skelton, R.E. (2000) A convexifying algorithm for the design of structured linear controllers. In:Proc.39th IEEE Conference on Decision and Control, Sydney , 2781-2786.

Orukpe, P.E., Jaimoukha, I.M. \& El-Zobaidi, H.M.H. (2007) Model Predictive Control Based on Mixed $\mathrm{H}_{2} / \mathrm{H}_{\infty}$ Control Approach, In:Proc. ACC, New York July, 2007, CD-ROM.

Peaucelle, D., Arzelier, D., Bachelier, O. \& Bernussou, J. (2000) A new robust D-stability condition for real convex polytopic uncertainty, Systems and Control Letters, 40, 21-30.

delaPena, D.M., Alamo, T., Ramirez, T. \& Camacho E. (2005) Min-max Model Predictive Control as a Quadratic Program, In: Proc. of 16th IFAC World Congress, Praga, CD-ROM.

Polak, E. \& Yang, T.H. (1993) Moving horizon control of linear systems with input saturation and plant uncertainty, Int. J. Control , 53, 613-638.

Rawlings, J. \& Muske, K. (1993) The stability of constrained Receding Horizon Control. IEEE Trans. on Automatic Control 38, 1512-1516.

Rohal-Ilkiv, B. (2004) A note on calculation of polytopic invariant and feasible sets for linear continuous -time systems. Annual Rewiew in Control , 28, 59-64.

Rosinová, D., Veselý, V. \& Kučera, V. (2003) A necessary and sufficient condition for static output feedback stabilizability of linear discrete-time systems, Kybernetika, Vol39, N4, 447-459.

Rossiter, J.A. (2003) Model Based Predictive Control: A Practical Approach,Control Series.

Veselý, V., Rosinová, D. \& Foltin, M. (2010) Robust model predictive control design with input constraints. ISA Transactions,49, 114-120.

Veselý, V. \& Rosinová, D. (2009) Robust output model predictive control design : BMI approach, IJICIC Int. Vol 5, 4, 1115-1123.

Yanou, A., Inoue,A., Deng, M. \& Masuda,S. (2008) An Extension of two Degree-of-freedom of Generalized Predictive Control for M-input M-output Systems Based on State Space Approach IJICIC, Vol4, N12, 3307-3318.

Zafiriou, E. \& Marchal, A. (1991) Stability of SISO quadratic dynamic matrix control with hard output constraints, AIChE J. 37, 1550-1560.

Wang, Z., Chen, Z. Sun, Q. \& Yuan, Z.(2006) GPC design technique based on MQFT for MIMO uncertain system, Int. J. of Innovitive Computing, Information and Control, Vol2, N3, 519-526.

Zheng, Z.Q. \& Morari, M. (1993) Robust Stability of Constrained Model Predictive Control, In Proc. ACC, San Francisco, CA, 379-383. 


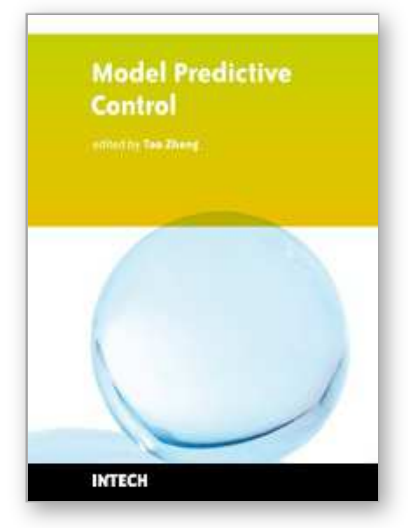

\author{
Model Predictive Control \\ Edited by Tao Zheng
}

ISBN 978-953-307-102-2

Hard cover, 304 pages

Publisher Sciyo

Published online 18, August, 2010

Published in print edition August, 2010

Frontiers of Model Predictive Control Robust Model Predictive Control Nonlinear Model Predictive Control Excellent Applications Guide for Researchers and Engineers Recent Achievements of Authors over the World Theory with Practical Examples Kinds of Algorithms for Choice

\title{
How to reference
}

In order to correctly reference this scholarly work, feel free to copy and paste the following:

Vojtech Vesely and Danica Rosinova (2010). Robust Model Predictive Control Design, Model Predictive Control, Tao Zheng (Ed.), ISBN: 978-953-307-102-2, InTech, Available from:

http://www.intechopen.com/books/model-predictive-control/robust-model-predictive-control-design

\section{INTECH}

open science | open minds

\section{InTech Europe}

University Campus STeP Ri

Slavka Krautzeka 83/A

51000 Rijeka, Croatia

Phone: +385 (51) 770447

Fax: +385 (51) 686166

www.intechopen.com

\section{InTech China}

Unit 405, Office Block, Hotel Equatorial Shanghai

No.65, Yan An Road (West), Shanghai, 200040, China

中国上海市延安西路65号上海国际贵都大饭店办公楼405单元

Phone: +86-21-62489820

Fax: $+86-21-62489821$ 
(C) 2010 The Author(s). Licensee IntechOpen. This chapter is distributed under the terms of the Creative Commons Attribution-NonCommercialShareAlike-3.0 License, which permits use, distribution and reproduction for non-commercial purposes, provided the original is properly cited and derivative works building on this content are distributed under the same license. 